\title{
MiR-410 Acts as a Tumor Suppressor in Estrogen Receptor-Positive Breast Cancer Cells by Directly Targeting ERLIN2 via the ERS Pathway
}

\author{
Hewen Wua,b Junli Lia En'en Guo ${ }^{a}$ Suxia Luo ${ }^{\mathrm{b}}$ Guohui Wang ${ }^{\mathrm{b}, \mathrm{c}}$

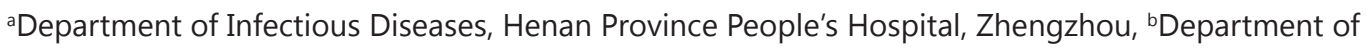

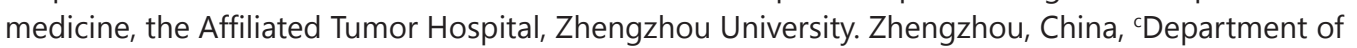

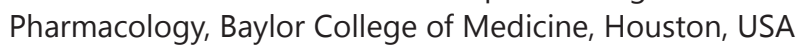

\section{Key Words.}

Micrornas .miRNAs) • MiR-410 • ERLIN2 • Tumorigenesis • Estrogen receptor

\begin{abstract}
.

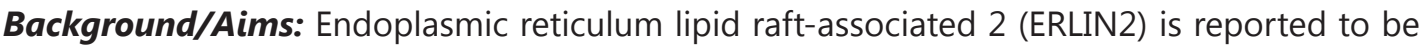

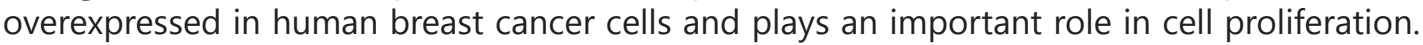

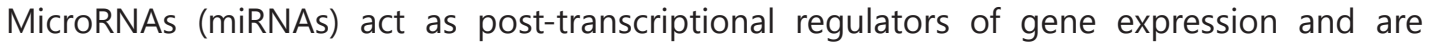

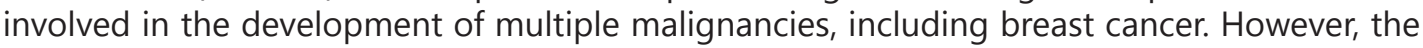

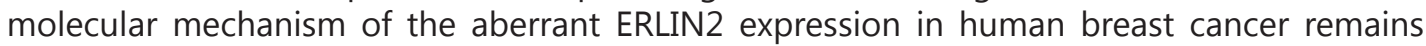

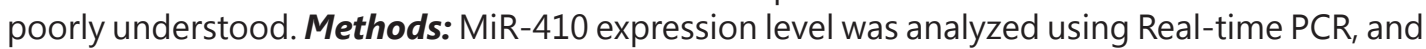

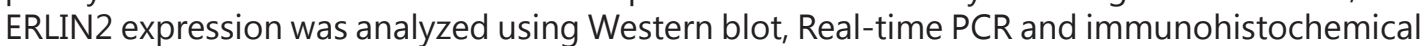

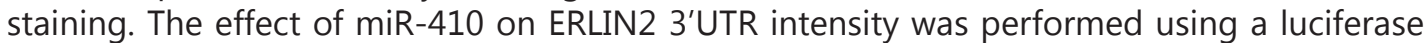

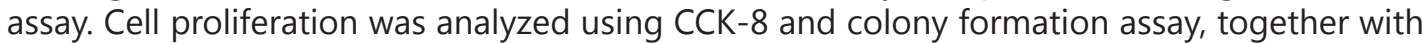

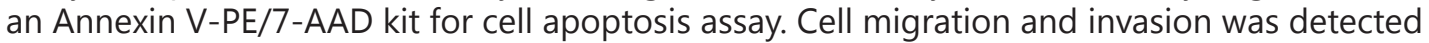

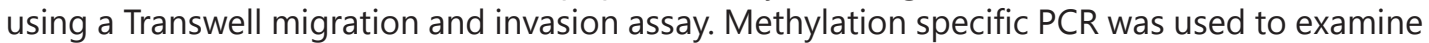

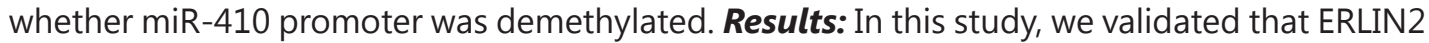

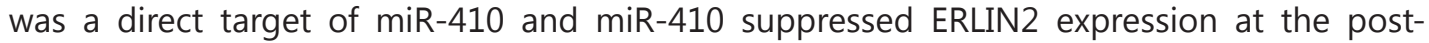

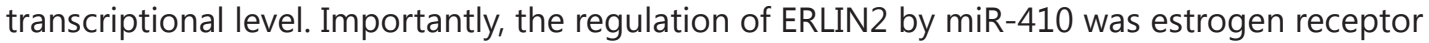

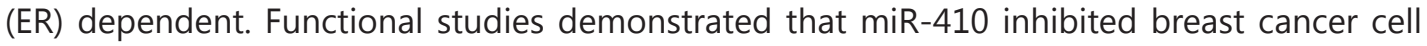

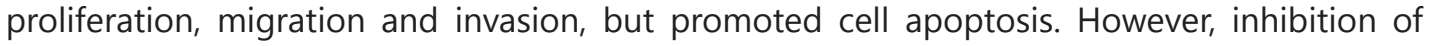
miR-410 resulted in opposite effects. A xenograft nude mouse model further confirmed that miR-410 suppressed breast tumor growth. In addition, miR-410 modulated the expression levels of epithelial-mesenchymal transition (EMT)-related genes. ERLIN2 knockdown suppressed cell proliferation, migration and invasion, as well as EMT. ERLIN2 overexpression can restore the cell proliferation, migration and invasion that were inhibited by miR-410. Furthermore, our data demonstrated that miR-410 inhibition suppressed the expression of
\end{abstract}


Wu et al.: the Act of MiR-410 on ERLIN2 in ER(+) Breast Cancer

endoplasmic reticulum-stress (ERS)-related genes, while ERLIN2 knockdown abrogated the effects of miR-410 inhibitor. Finally, we showed that miR-410 was downregulated in human ER-positive breast cancer tissues, inversely correlated with ERLIN2. We further demonstrated the downregulation of miR-410 in breast cancer might be due to the hypermethylation of its promoter. Conclusions: Our study indicates that miR-410 suppresses cell growth, migration and invasion by directly downregulating ERLIN2 in ER positive breast cancer, acting as a tumor suppressor. Our study also suggests that miR-410 may serve as a potential therapeutic target for patients with ER positive breast cancer.

\section{Introduction}

Breast cancer is the most common malignancy in women and represents the second cause of cancer-related mortality in women in the developing world, with the increasing incidence and mortality rate $[1,2]$. A previous study shows that approximately $70 \%$ of human breast cancer is estrogen receptor $+(E R+)$ and estrogen exerts significant roles in the breast tumorigenesis [3]. There are multiple genetic alternations in breast cancer. Studies indicate that the amplification of $8 p 11-12$ genomic region exists in $15 \%$ of breast cancers and contains several candidate oncogenes related to the survival and distant recurrence of the breast cancer patients. Endoplasmic reticulum lipid raft-associated 2 (ERLIN2) has been reported to serve as a candidate oncogene within the 8p11-12 amplicon and influences the transformed phenotypes [4-6]. A previous study shows that ERLIN2 is overexpressed in human breast cancer cells and tissue samples, and promotes cell survival through modulating the ER stress pathway [7]. Therefore, there is urgent need to elucidate the molecular mechanism underlying the aberrant expression of ERLIN2 in breast cancer, which will facilitate to explore and validate a novel potential therapeutic strategy for breast cancer patients.

MicroRNAs (miRNAs) are a conserved family of endogenous, small non-coding RNA molecules that are approximately $22 \mathrm{nt}$ in length and act as significant post-transcriptional regulators of gene expression by base paring with the 3'untranslated region (3'UTR) of target genes, resulting in the cleavage of target mRNAs or the repression of translation [8, 9]. MiRNAs are involved in multiple biological and pathological processes, including cell proliferation, cell apoptosis and metastasis $[10,11]$. They function as tumor suppressors or oncogenes depending on the roles of their targets [12, 13]. Accumulating evidence demonstrates that several miRNAs are deregulated in human breast cancer $[14,15]$. Dozens of miRNAs, such as miR-449a [16], miR-206 [17] and miR-26 [18] play crucial roles in the pathogenesis of breast cancer, serving as tumor suppressors or oncogenes.

In the current study, we aimed to validate a specific miRNA targeting ERLIN2 for elucidating the molecular mechanism of the aberrant ERLIN2 expression, and investigated its roles in the pathogenesis of breast cancer. We identified that ERLIN2 was a direct target of miR-410 and miR-410 suppressed ERLIN2 expression at the post-transcriptional level. MiR-410 suppressed breast cancer cell growth, migration and invasion, but promoted cell apoptosis through the downregulation of ERLIN2. MiR-410 and ERLIN2 were inversely correlated in ER positive breast cancer. Moreover, ERLIN2 knockdown can abrogate the inhibitory effects of miR-410 inhibitor on the ERS signaling pathway. Finally, we found that downregulation of miR-410 may be due to the hypermethylation of its promoter region.

\section{Materials and Methods}

\section{Human breast cancer tissue samples}

Breast cancer and adjacent non-tumor tissues were acquired from patients in the affiliated tumor hospital of Zhengzhou University, which were confirmed by the immunohistochemical staining. All breast cancer tissues were ER positive. The study was approved by the Ethical Committee of the affiliated tumor 


\section{Cellular Physiology Cell Physiol Biochem 2018;48:461-474 \begin{tabular}{c|c} 
DOI: 10.1159/000491777 & $\begin{array}{l}\text { O 2018 The Author(s). Published by S. Karger AG, Basel } \\
\text { www.karger.com/cpb }\end{array}$
\end{tabular} \\ Wu et al.: the Act of MiR-410 on ERLIN2 in ER(+) Breast Cancer}

hospital of Zhengzhou University and obtained the consent of patients for the use in research. The tissue samples were stored and frozen at $-80^{\circ} \mathrm{C}$ until use.

\section{Cell culture and transfections}

Human breast cancer cell lines, MCF-7, T47D (ER+) and MDA-MB-231 (ER-), were cultured in RPMI 1640 (Sigma, USA), supplemented with 10\% fetal bovine serum (FBS), $100 \mu \mathrm{g} / \mathrm{ml}$ streptomycin and $100 \mathrm{U} /$ $\mathrm{ml}$ penicillin. The cells were maintained in a humidified incubator at $37^{\circ} \mathrm{C}$ with $5 \% \mathrm{CO}_{2}$. For the treatment of $17 \beta$-estradiol (E2) in ER- breast cancer cells, the cells were incubated in phenol red-free RPMI 1640 with $5 \%$ dextran-coated charcoal-treated serum for at least 3 days before E2 $\left(10^{-8} \mathrm{~mol} / \mathrm{l}\right)$ treatment.

MiR-410 mimics, mimics control, miR-410 inhibitor and inhibitor control were purchased from GenePharma and were introduced to the cells using Lipofectamine ${ }^{\mathrm{TM}} 2000$ reagent (Invitrogen) according to the manufacturer's protocols.

\section{RNA isolation and real-time PCR}

Total RNAs (inclusive of miRNAs) were extracted from the transfected cells or tissue samples using the Trizol reagent according to the manufacturer's instructions. RNAs were quantified using the NanoDrop ND-2000 spectrophotometer. For the cDNA synthesis of ERLIN2, 500 ng of RNA was reversely transcribed using Reverse transcriptase, and Oligo $\mathrm{d}(\mathrm{T})$ was used as the common primer, while for the cDNA synthesis of miR-410, specific miR-410 reverse transcription primer was used. Real-time PCR was performed using the SYBR ${ }^{\mathrm{R}}$ Green Realtime PCR Master Mix (TOYOBO) on an ABI 7300 system according to the following procedures: $95^{\circ} \mathrm{C} 4 \mathrm{~min}$, followed by 40 cycles of $95^{\circ} \mathrm{C} 1 \mathrm{~min}, 58^{\circ} \mathrm{C} 30 \mathrm{sec}$ and $72^{\circ} \mathrm{C} 30 \mathrm{sec}$. U6 snRNA was employed as an internal control to normalize miR-410 expression. $\beta$-actin was used as an internal control to normalize ERLIN2 expression. The primers for reverse transcription (RT) and PCR were listed as follows: miR-410 RT primer: 5'-GTCGTATCCAGTGCAGGGTCCGAGGTATTCGCACTGGATACGACACAGGCCA-3'. U6 RT primer: 5'-GTCGTATCCAGTGCAGGGTCCGAGGTATTCGCACTGGATACGACAAAAATAT-3'. MiR-410 Forward: 5'-GTCAGCGCAATATAACACAG-3'. U6 Forward: 5'-GTCAGCGCGTGCTCGCTTCG-3'. Common reverse primer: 5'-GTGCAGGGTCCGAGGT-3'. $\beta$-actin: Forward: 5'-CTTAGTTGCGTTACA CCCTTTCTTG-3'; Reverse: 5'-CTTAGTTGCGTTACACCCTTTCTTG-3'. ERLIN2 Forward: 5'-TCCACCACGAACTGAACCAG-3'; Reverse: 5'-AACAGCTCAATGTAGACCTCTTG-3'.

In addition, miR-410 expression in cell colonies were also determined.

\section{Western blot assay}

Proteins were extracted from the transfected cells to examine ERLIN2 protein expression. Briefly, cells were lysed using RIPA buffer (50 mM Tris- $\mathrm{HCl}$, pH 8.8, $150 \mathrm{mM} \mathrm{NaCl}, 1 \% \mathrm{NP}-40,1 \%$ sodium deoxycholate, $0.1 \% \mathrm{SDS}$ ) for $30 \mathrm{~min}$ at $4^{\circ} \mathrm{C}$. Protein concentration was measured using a BCA assay and $50 \mu \mathrm{g}$ of proteins were then separated on a SDS-polyacrylamide gel electrophoresis (SDS-PAGE). Rabbit polyclonal antibody to ERLIN2 (Abcam, 1:1000), rabbit polyclonal antibody to E-cadherin, rabbit polyclonal antibody to vimentin, Snail and Fibronectin (1:1000, Abcam) and $\beta$-tubulin were used as primary antibodies. Horseradish Peroxidase-conjugated goat anti-rabbit IgG was used as a secondary antibody. The bands were detected using the ECL Western blotting kit according to the manufacturer's protocols. $\beta$-tubulin was used as a loading control.

\section{Plasmid construct and Luciferase assay}

ERLIN2 3'UTR containing the miR-410 binding site or the mutant ERLIN2 3'UTR (several bases within the binding site were mutated) were synthesized by annealing and then cloned into the PmeI and Xbal sites of the pmirGLO vector. The primers for the constructs were shown as followed: ERLIN2 3'UTR Top: 5'-AAACTA GCGGCCGC TAGT TTTATTAATAATTTTTATATAT-3'; Bottom: 5'-CTAGATATATAAAAATTATTAATAAAACTAGCGGCCGCTAGTTT-3'. Mutant ERLIN2 3'UTR Top: 5'-AAACTAGCGGCCGCTAGTTTTATTAATAATTTTAAAAAAT-3'; Bottom: 5'-CTAGATTTTTTAAAATTATTAATAAAACTA GCGGCCGCTAGTTT-3'.

For the luciferase assay, breast cancer cells were co-transfected with miR-410 mimics and wildtype or mutant luciferase constructs, together with controls. At $48 \mathrm{hrs}$ after transfection, the cells were collected and subjected to luciferase assay using a Dual-Glo Luciferase Assay System (Promega) according to the 


\section{Cellular Physiology Cell Physiol Biochem 2018;48:461-474 \begin{tabular}{ll|l} 
DOI: 10.1159/000491777, & $\begin{array}{l}\text { O 2018 The Author(s). Published by S. Karger AG, Basel } \\
\text { www.karger.com/cpb }\end{array}$
\end{tabular} \\ Wu et al.: the Act of MiR-410 on ERLIN2 in ER(+) Breast Cancer}

manufacturer's instructions. The Renilla luciferase intensity was used as an internal control to normalize the firefly luciferase intensity.

\section{Cell Counting Kit-8 (CCK-8) assay}

Cell viability was tested using a CCK-8 assay according to the manufacturer's protocols. The cells transfected with miR-410 mimics or miR-410 ASO or controls for 48 hrs were planted at a density of 2000 cells/well in 96-well plates. At 12 hrs after the transfected cell inoculation, the cells were incubated with CCK-8 for $1 \mathrm{hr}$. The absorbance was measured at $450 \mathrm{~nm}\left(\mathrm{~A} 450_{\mathrm{nm}}\right)$ using a spectrophotometer.

\section{Colony formation assay}

The transfected cells were seeded in 12-well plates (150 cells/well) for the colony formation assay. The medium was changed every 3 days until most of the colonies contained more than 50 cells. The colonies were then washed with PBS and stained with $1 \%$ crystal violet.

\section{In vivo animal study}

A xenograft nude mouse model was established in 6 8-week-old NOD/SCID mice under specific pathogen-free conditions and approved by the Institutional Animal Care and Use Committee of the affiliated tumor hospital of Zhengzhou University. Briefly, $1 \times 10^{6}$ MCF-7 cells transfected with $50 \mathrm{nM}$ miR-410 agomir (RiboBio) or agomir control were suspended in $100 \mu \mathrm{l}$ of PBS and subcutaneously injected into the flank of the mice. On week 1,2 and 3, tumor volume was examined by measuring the length $(L)$ and width $(W)$ with calipers and calculated with the formula $\left(L \times W^{2}\right) / 2$. The tumor xenografts were used to determine miR-410 and ERLIN2 by real-time PCR and western blot.

\section{Transwell migration and invasion assays}

Cell migration and invasion were analyzed using a Transwell migration and invasion kit (BD Biosciences) according to the manufacturer's instructions. Briefly, $2.5 \times 10^{4}$ transfected breast cancer cells were suspended in $250 \mu \mathrm{l}$ of serum-free medium and then seeded in the upper chamber of the Transwell inserts for the migration and invasion assay. For the invasion assay, the upper chamber was firstly coated with matrigel. The lower chamber of the inserts was incubated with $10 \%$ FBS containing medium. After the cells migrated or invaded for $12 \mathrm{hrs}$ and $20 \mathrm{hrs}$, respectively, the cells migrating or invading into the membrane were fixed and stained with crystal violet, while the cells that did not migrate or invade into the membrane were scraped with cotton tips. The migrated or invaded cells were finally observed and taken pictures under a microscope.

\section{Cell apoptosis assay}

Cell apoptosis was examined using an Annexin V-PE/7-AAD kit and flow cytometric analysis according to the manufacturer's instructions as previously described [19]. The cells with Annexin V-PE $/ 7-\mathrm{AAD}^{-}$were calculated to represent apoptotic cells.

\section{Immunohistochemical staining}

Immunohistochemistry was performed on archival, formalin-fixed and paraffin-wax-embedded sections of human breast cancer tissues and adjacent non-tumor tissues according to the manufacturer's protocols. Rabbit monoclonal antibody to ERLIN2 (1:1000, Abcam) was used for the primary antibody.

\section{siRNA treatment}

Cells were transfected with siRNA against for ERLIN2 or scrambled controls at a final concentration of $100 \mathrm{nM}$. At $48 \mathrm{hrs}$ after transfection, the cells were collected and subjected to western blot assay to determine ERLIN2 protein expression. $\beta$-tubulin served as a loading control.

\section{5-Aza treatment}

The demethylation reagent 5-Aza (5-Aza-2'-deoxycytidine) was used to detect whether the downregulation of miR-410 was related to the promoter hypermethylation. The breast cancer cells were seeded into 6-well plates and then treated with $1 \mu \mathrm{mol} / \mathrm{L}$ 5-Aza for approximately $96 \mathrm{hrs}$. The cells were finally harvested and subjected to real-time PCR to analyze miR-410 expression level. 


\section{Cellular Physiology Cell Physiol Biochem 2018;48:461-474 \begin{tabular}{c|c} 
DOI: 10.1159/000491777 & $\begin{array}{l}\text { O 2018 The Author(s). Published by S. Karger AG, Basel } \\
\text { www.karger.com/cpb }\end{array}$
\end{tabular}}

\section{Methylation specific PCR (MSP)}

Genomic DNA was extracted from the cultured breast cancer cells using a cultured cell extraction kit (ABigen) in accord with the manufacturer's instructions. Subsequently, sodium bisulfite modification was applied to the genomic DNA using an EZ Methylation Kit (Zymo Research), followed by MSP using primers specific to methylated or unmethylated miR-410 promoter. MethPrimer software was used to design MSP primers and the primers were shown as follows: methylated primers: Forward: 5'-GTTTTTTTGAGGGTAGGAGTAGGAC-3'; Reverse: 5'-AAATACCATCGACTCAAAAACGTA-3'; unmethylated primers: Forward:5'-GTTTTTTTGAGGGTAGGAGTAGGAT-3'; Reverse: 5'-AAATACCATCAACTCAAAAACATA-3'.

\section{Statistical analysis}

The data were represented as mean \pm SD (standard variation) from three independent experiments. The statistical analysis was performed using GraphPad Prism 5 software. The differences between two groups were analyzed using the two-tailed Students' $t$-test. For the differences among three groups, oneway ANOVA followed by a LSD (least significant difference) test was used. A value of $p<0.05$ was considered statistically significant.

\section{Results}

\section{ERLIN2 is a direct target of miR-410 in human breast cancer cells}

Three algorithms (PicTar, microRNA.org and TargetScan) were used to predict miRNAs which may target ERLIN2. Among the candidate miRNAs, 9 miRNAs (miR-543, miR-3423p, miR-339-5p, miR-216b, miR-590-3p, miR-181b/d, miR-410, miR-433 and miR-196a/b) were selected for further study. Next, we checked the effect of these candidate miRNAs on ERLIN2 expression by Western blot and Real-time PCR in MCF-7 and MDA-MB-231 cell lines. Among these miRNAs, we found that miR-410 significantly reduced ERLIN2 protein level in MCF-7 cells. In addition, miR-433, miR-181b/d and miR-343-3p also suppressed ERLIN2 protein levels. However, none of miRNAs downregulated ERLIN2 expression in MDA-MB-231 cells (Fig. 1A). In line with the results from Western blot assay, we found that only miR-410 inhibited ERLIN2 mRNA expression in MCF-7 cells, but not in MDA-MB-231 cells (Fig. 1B). Moreover, we detected miR-410 and ERLIN2 expression in five breast cell lines, MCF-7, MDAMB-231, MDA-MB-453, T47D and MDA-MB-435s. We found that miR-410 was significantly downregulated in T47D cells compared to MCF-7 cells, with a slight decrease in MDA-MB435s cells (Fig. 1C). However, ERLIN2 protein level was overexpressed in these cell lines (Fig. 1D), suggesting a negative relationship between miR-410 and ERLIN2 in MCF-7 and T47D cells, except in MDA-MD-231 and MDA-MB-453 cells.

To determine whether ERLIN2 is a direct target of miR-410 in breast cancer, a luciferase reporter construct was first generated. ERLIN2 3'UTR containing wildtype or mutant miR410 binding sites was cloned and inserted downstream of a luciferase reporter gene (Fig. $1 E$ ), and subsequently co-transfected MCF-7 cells with miR-410 or control. As shown in Fig. $1 \mathrm{~F}$, we found that miR-410 reduced the luciferase intensity controlled by ERLIN2 3'UTR, while miR-410 inhibition increased ERLIN2 3'UTR intensity. However, the inhibitory effect of miR-410 on ERLIN2 3'UTR was abrogated when the binding sites were mutated. Taken together, the results indicate that miR-410 downregulates ERLIN2 expression by directly binding to its 3'UTR.

The regulation of ERLIN2 by miR-410 is estrogen-dependent in breast cancer cells

The above results showed that miR-410 inhibited ERLIN2 expression in MCF-7 cells but not in MDA-MB-231 cells, implying that the regulation of ERLIN2 by miR-410 may be related to estrogen. We then detected the effect of miR-410 on ERLIN2 expression in other ER (-) cells, MDA-MB-453 and MDA-MB-435s. As shown in Fig. 2A, miR-410 did not influence ERLIN2 protein level in MDA-MB-453 and MDA-MB-435s. Next, we aimed to investigate whether estrogen affected the expression of miR-410. Results from Fig. 2B showed that miR- 
Fig. 1. ERLIN2 is a direct target of miR-410 in human breast cancer. (A) ERLIN2 protein level was analyzed by Western blot in MCF-7 and MDA-MB-231 cells transfected with different miRNA mimics or mimics control. The graph represented relative ERLIN2 protein levels normalized to the control. $\beta$-tubulin was used as a loading control. ${ }^{*} \mathrm{P}<0.05$. ${ }^{* *} \mathrm{P}<0.01$. (B) ERLIN2 mRNA level was analyzed by real time PCR in MCF-7 and MDA-MB-231 cells transfected with different miRNA mimics or mimics control. $\beta$-actin was used as an endogenous control. $* \mathrm{P}<0.05$. (C) miR-410 expression level was analyzed by real time PCR in a series of cell lines, including MCF-7, MDA-MB-231, MDA-MB-453, T47D and MDA-MB-435s. ${ }^{*} \mathrm{P}<0.05$. (D) ERLIN2 protein level was analyzed in five cell lines, MCF-7, MDA-MB-231, MDA-MB-453, T47D and MDA-MB-435s. (E) There was a miR-410binding site on ERLIN2 3'UTR. The line showed the complementary base pairs between miR-410 and ERLIN2 3'UTR. The bases in red color represented the mutant based within the binding site. Either the wild type or mutant ERIN2 3'UTR containing miR-410 binding site was cloned downstream of an opening reading frame in a luciferase reporter, pmirGLO plasmid. (F) MCF-7 cells were co-transfected with either miR-410 or miR-410 inhibitor and wild type or mutant luciferase reporter constructor, together with control. At $48 \mathrm{hrs}$ after transfection, the cells were harvest and subjected to luciferase reporter assay to examine the effect of miR-410 on ERLIN2 3'UTR intensity. ${ }^{*} \mathrm{P}<0.05$. All data were from three independent experiments and one representative experiment was shown in the manuscript.

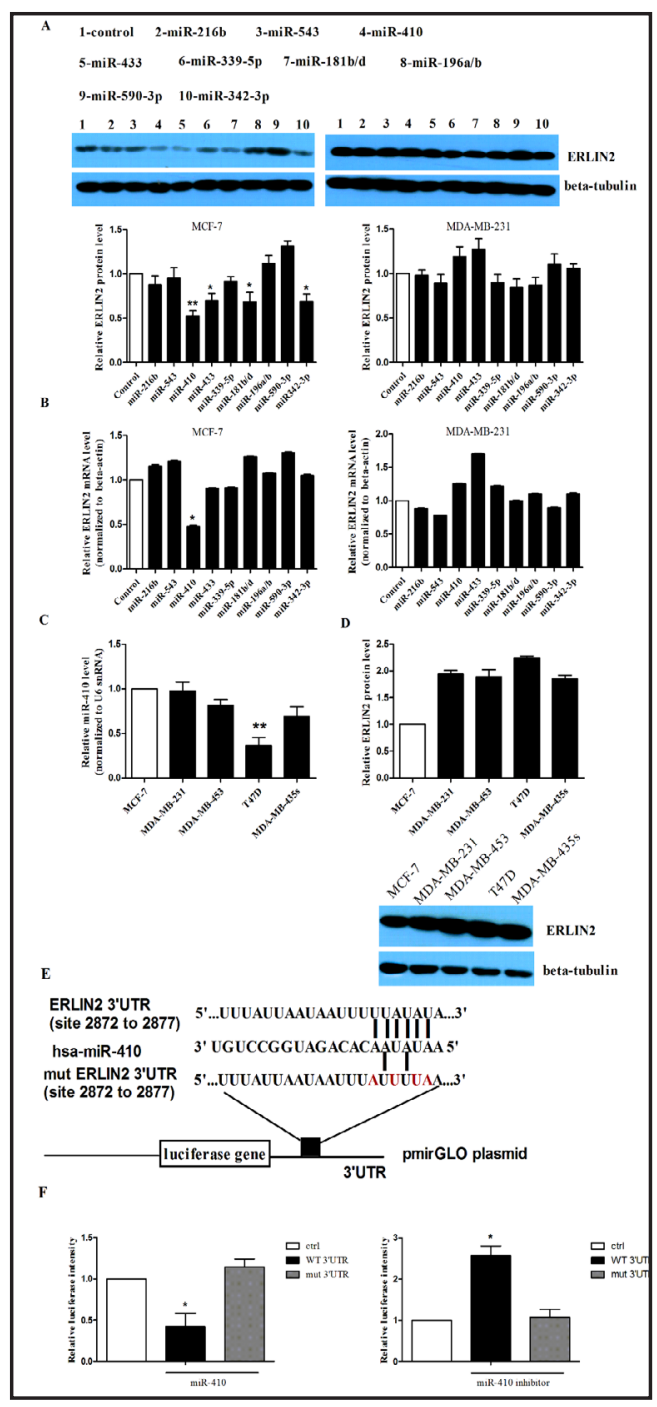

410 expression was not regulated by $17 \beta$-estradiol (E2). We furthermore found that miR410 suppressed ERLIN2 3'UTR intensity in MDA-MB-231 and MDA-MB-453 cells when the cells were treated with E2 (Fig. 2C). However, when MCF-7 and T47D cells were treated with the inhibitor of estrogen, the inhibitory effect of miR-410 on ERLIN2 intensity was partially abrogated (Fig. 2D). Overall, the data indicate that the regulation of ERLIN2 by miR-410 is estrogen-dependent.

\section{MiR-410 suppresses breast cancer cell growth but promotes cell apoptosis}

We next investigated the effects of miR-410 on the malignant phenotypes in breast cancer cells. CCK-8 assay showed that miR-410 inhibition enhanced the cell viability in MCF-7 cells compared to cells transfected with controls. In contrast, the cell viability was inhibited when treated with miR-410 mimics. Similar results were obtained in T47D cells (Fig. 3A). In line with the results from CCK-8 assay, we found that miR-410 inhibition increased the number of colonies in MCF-7 cells, while miR-410 overexpression reduced the colony number in T47D cells (Fig. 3B). The miR-410 expression in the colonies was also determined by qPCR (Fig 3B). To explore whether the effects of miR-410 on cell growth is related to cell apoptosis, we performed apoptosis assay via flow cytometry. As shown in Fig. 3C, the apoptosis rate was suppressed when the cells were transfected with miR-410 inhibitor. On the contrary, the apoptotic rate was increased when transfected with miR-410

\section{KARGER}


Fig. 2. The regulation of ERLIN2 by miR-410 is estrogen dependent. (A) ERLIN2 protein level was analyzed by Western blot in estrogen-negative cell lines after transfection with miR-410 or control. $\beta$-tubulin was used as a loading control. The graph represented the relative ERLIN2 protein level. (B) miR-410 expression level was analyzed by real time PCR in MCF-7 and MDA-MB-231 cells treated with or without $17 \beta$-estradiol (E2) at different time points. (C-D) miR-410 expression level was analyzed by real time PCR in MDA-MB-231 (C) or MCF-7(D) cells treated with E2 (C) or E2 inhibitor (D), respectively. ${ }^{*} \mathrm{P}<0.05$. All data were from three independent experiments and one representative experiment was shown in the manuscript.

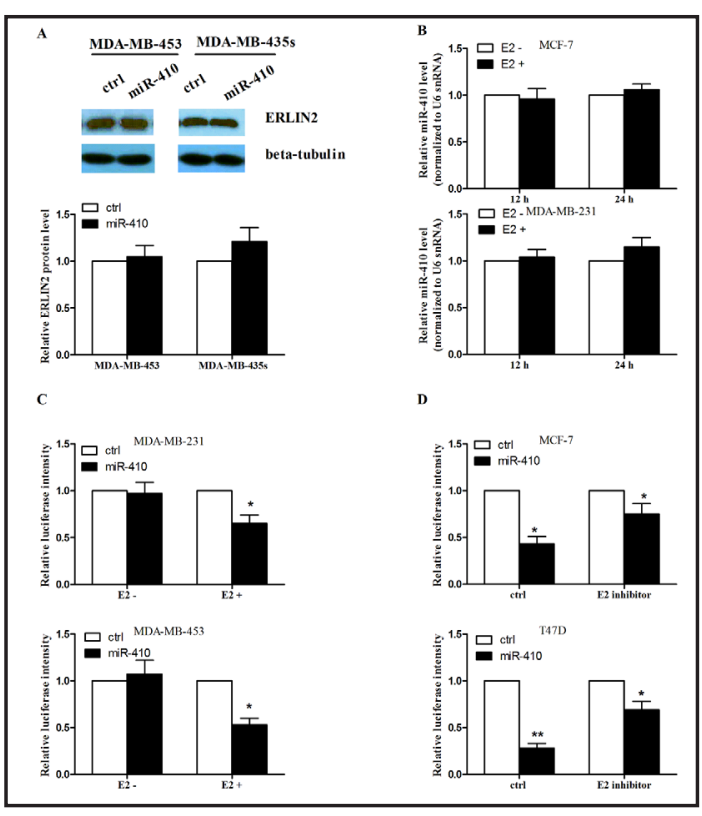

Fig. 3. miR-410 suppresses cell growth but enhances cell apoptosis in human breast cancer. (A-B) The effect of miR-410 on cell growth was examined by cell viability (A) and colony formation assays (B) in breast cancer cells transfected with miR-410 inhibitor or miR410 mimics. Meanwhile, miR-410 expression was determined by qPCR in the cell colonies with miR-410 mimics or miR-410 inhibitor. ${ }^{*} \mathrm{P}<0.05$. ${ }^{* *} \mathrm{P}<0.01$. (CD) Cell apoptosis was analyzed by flow cytometry using an Annexin V-PE/7-AAD assay in MCF-7 (C) and T47D (D) cells transfected with miR-410 inhibitor or miR410 mimics. ${ }^{*} \mathrm{P}<0.05$. ${ }^{*} \mathrm{P}<0.01$. The $y$-axis represented the fluorescence intensity of cells stained with 7-AAD (staining of nucleus), while $\mathrm{x}$-axis represented

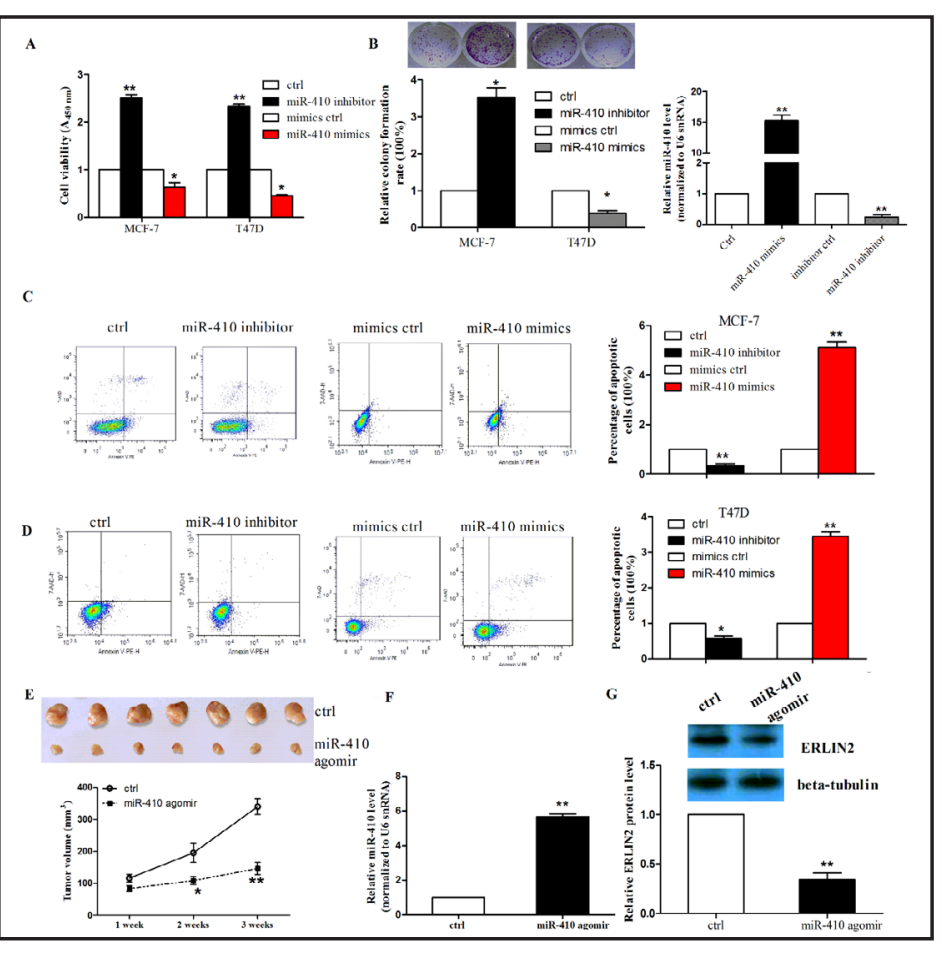
the fluorescence intensity of cells with Annexin V-PE (staining of cell membrane). 7-AAD-/Annexin V-PE+: apoptotic cells (lower right); 7-AAD-/Annexin V-PE-: live cells (lower left); 7-AAD+/Annexin V-PE-: dead cells (upper left); 7-AAD+/ Annexin V-PE+: late apoptotic cells (upper right). (E) In vivo animal study showed that miR-410 suppressed tumor growth. The image represented the tumor xenografts formed by miR-410 agomir-transfected cells or control cells. ${ }^{*} \mathrm{P}<0.05$. ${ }^{* *} \mathrm{P}<0.01$. (F) miR-410 expression level was analyzed by real time PCR in the xenografts. ${ }^{* *} \mathrm{P}<0.01$. Each group had 7 xenograft samples. (G) ERLIN2 protein level was analyzed by Western blot in the xenografts treated with miR-410-transfected cells or control cells. Each group had 7 xenograft samples. $\beta$-tubulin was used as a loading control. Relative protein levels were analyzed shown in the graph. All data were from three independent experiments and one representative experiment was shown in the manuscript. 
mimics. Similar data were acquired in T47D cells (Fig. 3D). These data suggest that miR-410 suppresses cell proliferation via the promotion of cell apoptosis. In addition, a xenograft tumor mouse model was established by subcutaneously injecting miR-410 agomir-treated cells or cells with agomir control into the flank of the mouse. The xenograft volumes were measured on week 1, 2 and 3. As shown in Fig. 3E, we found that miR-410 agomir suppressed the xenograft volumes compared to control group. The expression of miR-410 was increased in the xenograft treated with miR-410 agomir, suggesting that the delivery of miR-410 agomir was successful (Fig. 3F). As expected, ERLIN2 protein expression was inhibited in the xenograft tissues treated with miR-410 agomir compared to control group (Fig. 3G).

\section{MiR-410 suppresses cell migration and invasion as well as EMT in breast cancer cells}

Besides the cell growth, we further detected the effects of miR-410 on cell migration and invasion. As shown in Fig. 4A, miR-410 inhibition increased the number of migratory cells compared to inhibitor control in MCF-7 cells, while the migratory cell number was reduced when the cells were transfected with miR-410 mimics. Similar results were observed in T47D cells transfected with miR-410 inhibitor or miR-410 mimics. In line with the results obtained in migration assay, we found that miR-410 inhibition increased the number of invading cells, while miR-410 overexpression resulted in a decrease in the number of invading cells (Fig. 4B). Considering epithelial-mesenchymal transition (EMT) is a basic cause of cell invasion and a characteristic feature of cells undergoing proliferation, we next examined the effects of miR-410 on EMT-related genes' expression. As shown in Fig. 4C, miR-410 inhibition increased the expression of Vimentin, Snail and Fibronectin but reduced the E-cadherin mRNA expression. In contrast, miR-410 resulted in opposite effects. Similar results were obtained in the Western blot assay (Fig. 4D). Taken together, the results indicate that miR410 inhibits cell migration and invasion via the suppression of EMT.

\section{ERLIN2 mediates the roles of miR-410 in the pathogenesis of breast cancer}

We aimed to determine whether ERLIN2 was a functional target of miR-410 in breast cancer cells. ERLIN2 knockdown or ERLIN2 overexpression was confirmed by Western blot assay (Fig. 5A and 5C). We found an increase in MCF-7 cell growth (Fig. 5A), migration and invasion (Fig. 5B) induced by miR-410 inhibition was restored when the cells were co-transfected ERLIN2 siRNA, compared to the cells with controls. However, ERLIN2

Fig. 4. miR-410 suppresses cell migration and invasion in human breast cancer. (A-B) Cell migration and invasion assays were performed in MCF-7 and T47D cells transfected with miR410 inhibitor or miR-410 mimics or corresponding controls. The images represented the migratory or invasive cells per field under a microscopy. ${ }^{*} \mathrm{P}<0.05$. ${ }^{* *} \mathrm{P}<0.01$. (CD) EMT-related markers (Vimentin, Snail, fibronectin, E-cadherin) were examined by real time PCR (C) and Western blot (D) in MCF-7 and T47D cells transfected with miR410 inhibitor or miR-410 mimics, respectively. Relative protein levels

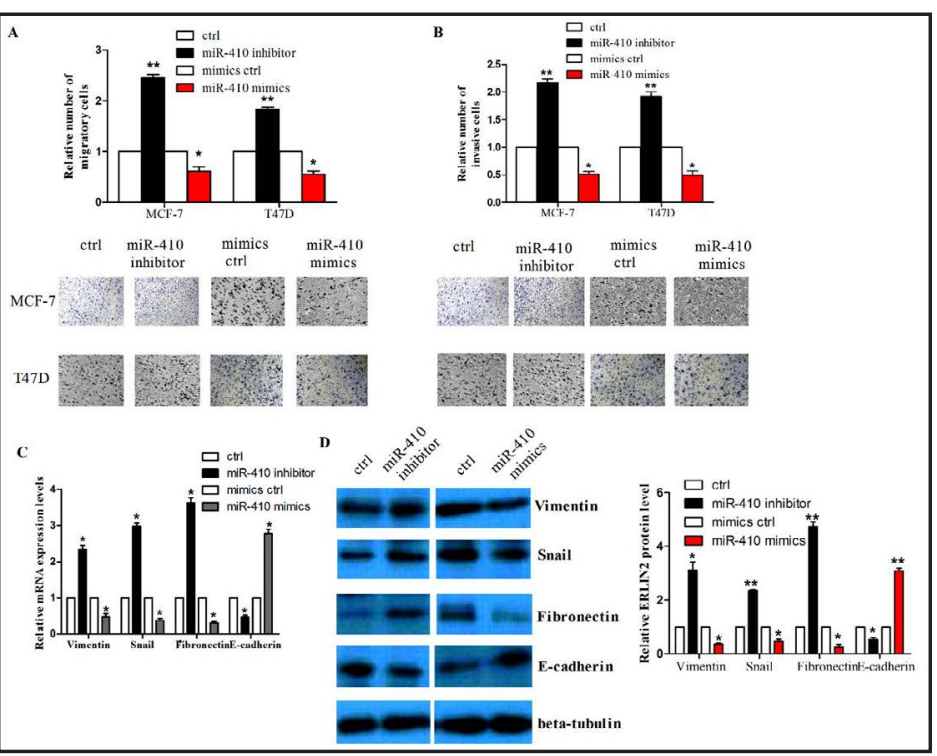
were analyzed shown in the graph. ${ }^{*} \mathrm{P}<0.05$. ${ }^{* *} \mathrm{P}<0.01$. All data were from three independent experiments and one representative experiment was shown in the manuscript.

\section{KARGER}


Fig. 5. The effect of miR-410 on cell growth, migration and invasion are abrogated when ERLIN2 is overexpressed. (A-B) ERLIN2 protein was confirmed by Western blot in MCF-7 cells. MCF-7 cells were co-transfected with miR-410 inhibitor and ERLIN2 siRNA, together with control. Cell proliferation (A) and migration and invasion assays (B) were then tested. ${ }^{*} \mathrm{P}<0.05$. (C-D) ERLIN2 protein was confirmed by Western blot in T47D cells. T47D cells were co-transfected with miR-410 mimics and ERLIN2overexpressing plasmid, together with control. Cell proliferation (C) and migration and invasion assays (D) were then tested. ${ }^{*} \mathrm{P}<0.05$. All data were from three independent experiments and one representative experiment was shown in the manuscript.

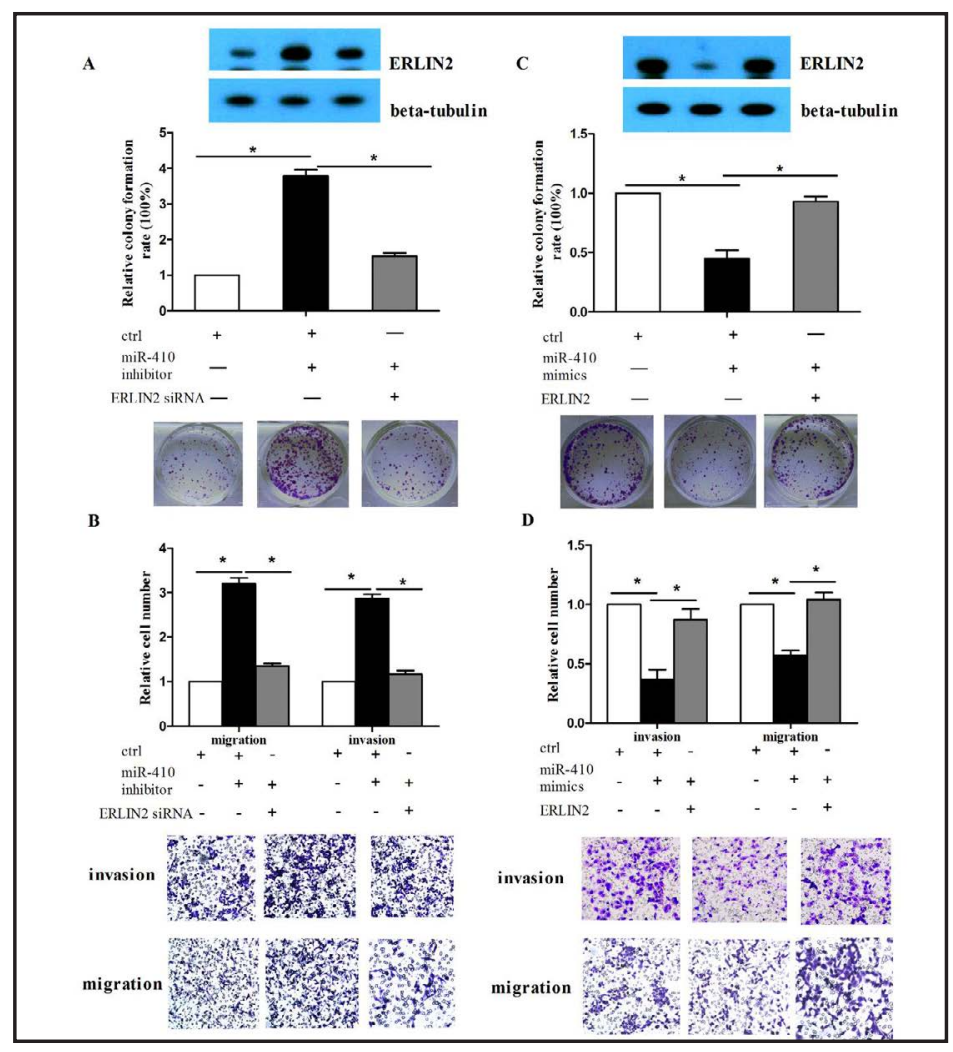

overexpression can attenuate the inhibitory effects of miR-410 on T47D cell growth (Fig. 5C), migration and invasion (Fig. 5D). These results suggest that miR-410 suppresses cell growth, migration and invasion in breast cancer cells partially through the downregulation of ERLIN2.

\section{MiR-410 and ERLIN2 are inversely correlated in breast cancer}

To further investigate the clinicopathological significance of the abnormal expression of miR-410 and ERLIN2, we analyzed miR-410 and ERLIN2 expression by real-time PCR in $\mathrm{ER}(+)$ breast cancer tissues and adjacent non-tumor tissues. As shown in Fig. 6A, we found that miR-410 was significantly downregulated in breast cancer tissues compared to adjacent non-tumor tissue samples, while ERLIN2 was upregulated in breast cancer tissue samples (Fig. 6B). In line with the real-time PCR results, immunohistochemical staining (Fig. 6C) showed that ERLIN2 was upregulated in breast cancer tissues.

MiR-410 and ERLIN2 can modulate the expression of ERS-related proteins in breast cancer

Since the ERS signaling pathway plays important roles in the pathogenesis of cancers, we then determined the effects of miR-410 on the ERS-related molecules' expression by Western blot in MCF-7 and T47D cells. As show in Fig. 6D, we found that inhibition of miR-410 expression suppressed the expression of CHOP, GRP94, Bip as well as the phosphorylation level of eIF $2 \alpha$ in MCF-7 cells, but slight effect on phosphorylation of PERK. In addition, we also examined the levels of apoptosis-related protein and found that miR-410 inhibition increased Bcl-2 expression level. Importantly, ERLIN2 knockdown abrogated the effects of miR-410 inhibition on the expression of ERS signaling and Bcl-2 protein levels. However, miR-410 mimics led to opposite results compared to the cells with miR-410 inhibitor, and ERLIN2 overexpression can attenuate miR-410 inhibitor-induced effects on these proteins (Fig. 6D). 


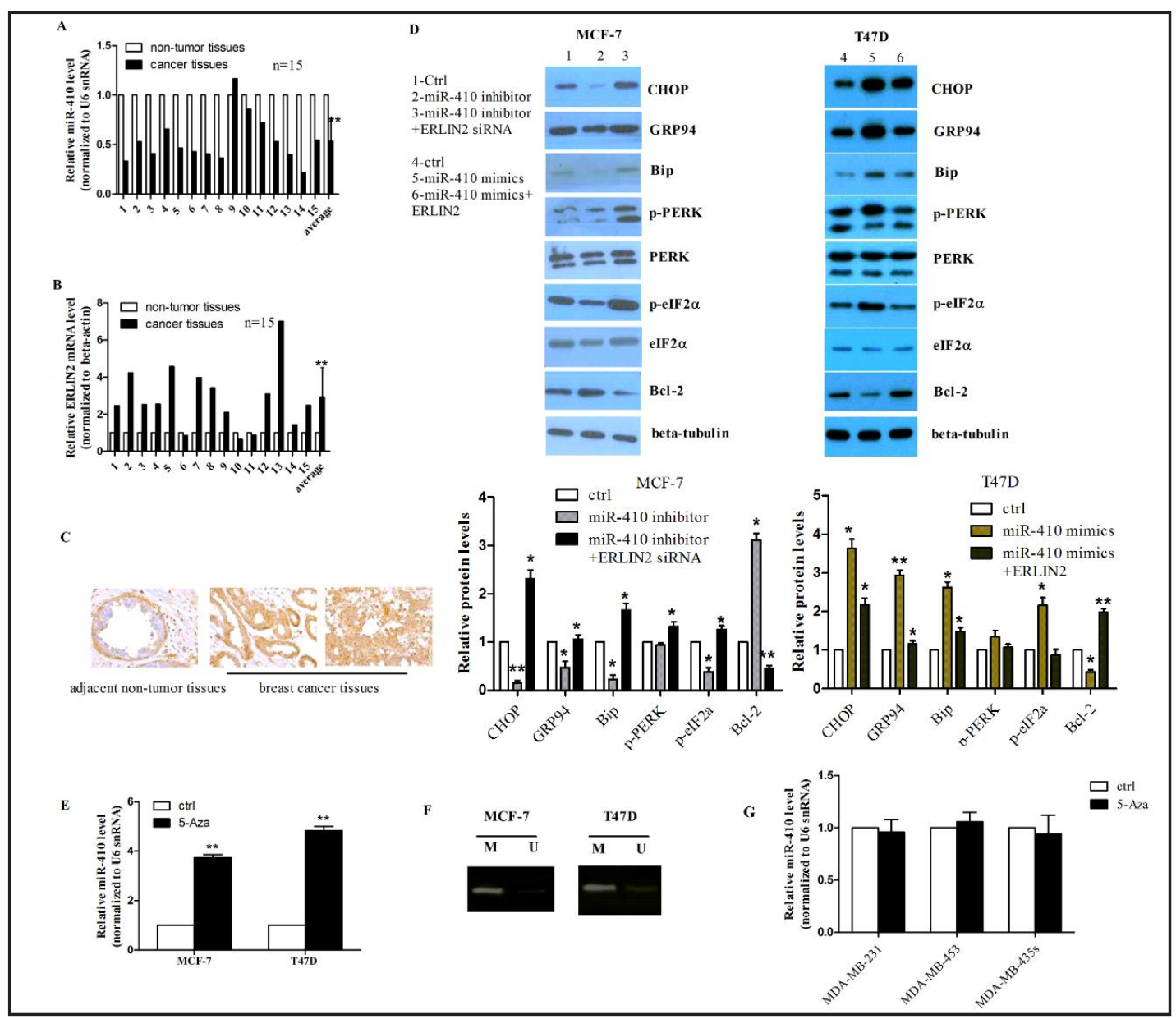

Fig. 6. MiR-410 and ERLIN2 are inversely expressed in human breast cancer tissues. (A) miR-410 expression was analyzed by real time PCR in 15 pairs of breast cancer tissues and adjacent non-tumor tissues. U6 RNA was used as an internal control. ${ }^{* *} \mathrm{P}<0.01$. (B-C) ERLIN2 protein levels were analyzed by Western blot (B) and immunohistochemical staining $(\mathrm{C})$ in breast cancer tissues and adjacent non-tumor tissues. $\beta$-tubulin was used as an internal control. (D) miR-410 and ERLIN2 modulates the expression of ERS pathway-related proteins in MCF-7 and T47D cells. Cells transfected with miR-410 inhibitor or miR-410 mimics or miR-410 inhibitor together with ERLIN2 siRNA or miR-410 mimics and ERLIN2 were subjected to Western blot to analyze the expression of ERS pathway-related proteins, such as CHOP, GRP94, Bip, PERK and eIF2a, as well as $\mathrm{BCl}-2$. Relative protein levels were analyzed shown in the graph. ${ }^{*} \mathrm{P}<0.05$. ${ }^{*} * \mathrm{P}<0.01$. (E-F) miR-410 is hypermethylated in human breast cancer. (E) miR-410 expression was analyzed by real time PCR in MCF7 and T47D cells after treated with 5-Aza, together with control. U6 RNA was used as an internal control. ${ }^{* *} \mathrm{P}<0.01$. (F) Methylation specific PCR was used to detect whether the promoter region of miR-410 was methylated. All data were from three independent experiments and one representative experiment was shown in the manuscript. (G) miR-410 expression was analyzed by qPCR in the ER(-) cell lines.

The downregulation of miR-410 in breast cancer may be related to DNA methylation

Finally, we aimed to investigate the potential mechanism of miR-410 downregulation in breast cancer. Results in a previous study indicate that there is a CpG island around the genomic locus of miR-410 [20]. In order to determine whether miR-410 promoter was regulated by CpG hypermethylation in ER(+) breast cancer, 5'-aza-2'-deoxycytidine (5-Aza) was added in MCF-7 and T47D cells. Real-time PCR results revealed that miR-410 expression was increased after 5-Aza treatment, compared to control groups (Fig. 6E). Moreover, methylation-specific PCR (MSP) was performed and we found that miR-410 was totally 
methylated in MCF-7 and T47D cells (Fig. 6F). However, miR-410 expression was not affect by 5-Aza in ER(-) cell lines, suggesting that it may be not methylated in ER(-) cells (Fig. 6G).

\section{Discussion}

The current study demonstrated that miR-410 suppressed cell growth, migration and invasion as well as EMT by downregulating ERLIN2 via the modulation of ERS-related genes, acting as a tumor suppressor in ER(+) breast cancer. The study also suggests that delivery of miR-410 has potential as a therapeutic strategy for breast cancer patients.

The roles of miR-410 in this study was in agree with the findings in other studies. For examples, miR-410 is downregulated in pituitaty gonadotroph tumors and suppresses cell proliferation by targeting CCNB1 [21]. MiR-410 inhibits cell growth, migration and invasion as well as the G1/S cell-cycle arrest through the downregulation of angiotensin II type 1 receptor in pancreatic cancer, functioning as a tumor suppressor [22]. In glioma, miR-410 suppresses the cell proliferation and invasion by targeting MET [23]. MiR-410 plays similar roles in gastric cancer by targeting MDM2, and downregulation of miR-410 is associated with lymph-node metastasis [24]. Moreover, miR-410 enhances the radiosensitivity of gastric cancer cells and suppresses cell proliferation as well as radio-induced EMT [25]. However, several data in the previous studies indicate that miR-410 acts as an oncogene in diverse cancers, such as NSCLC [20,26], liver and colorectal cancers [27]. In conclusion, the discrepancy for the roles of miR-410 in cancers may be due to the different targets of miR-410 in diverse tumors and its tissue-specificity, which meant different tumors have varied clinical and pathological characters. Besides tumors, miR-410 is also involved in the pathogenesis of system lupus erythematosus (SLE) by regulating IL-10 expression through targeting STAT3 [28].

ERLIN2 is been reported to be overexpressed in human breast cancer, and promoted cancer cell proliferation [4-7]. It is reported that ERLIN2 can interact with the mitosispromoting factor complex cyclinB1/cdk1, and stabilizes cyclinB1 via facilitating K63-linking ubiquitination. It can also enhance cell cycle progression, cell proliferation, malignancy and the cell response to anticancer drugs of breast cancer [29]. In agree with the findings in the previous studies, our study showed that ERLIN2 increases cell growth, migration and invasion that were inhibited by miR-410, while ERLIN2 knockdown resulted in opposite effects. In addition, we validated that ERLIN2 was a direct target of miR-410, and miR-410 downregulated ERLIN2 expression. In our study, we found ERLIN2 was highly expressed in ER(+) breast cancer tissues, while miR-410 was downregulated compared to adjacent nontumor tissues. Moreover, our data indicated that the regulation of ERLIN2 by miR-410 was estrogen-dependent. The inhibitory roles of miR-410 in ERLIN2 expression only existed in ER(+) breast cancer cell lines, not in ER- cell lines. However, when we treated $17 \beta$-estradiol (E2) into ER- breast cancer cells, we found that miR-410 can inhibit ERLIN2 expression. While we treated ER(+) cells with estrogen inhibitors, the downregulation of ERLIN2 by miR-410 was ameliorated. The above results were similar with the previous findings that the different regulatory roles of miRNAs in two breast cancer subtypes may be due to the different expression of several key miRNA processing genes between ER- and ER+ breast cancer, especially Ago 2 and Dicer [30, 31].

ERS pathway is involved in several biological processes, such as cell apoptosis. For example, extracts of Annona muricata can cause apoptosis of cancer cells and inhibit tumor growth through the activation of ERS pathway, with the upregulation of GRP94, HSP70, Bip and CHOP, as well as the upregulated phosphorylation of PERK and eIF2 $\alpha$ [32]. Andrographolide induces apoptosis of colon cancer cells via the upregulation of ERS proteins GRP78, CHOP as well as the decreased $\mathrm{BAX} / \mathrm{Bcl}-2$ ration [33]. ERS pathway is also reported to participate in the malignancy of diverse cancers, such as the metastasis of breast cancer [34, 35], the poor progression of gastric carcinoma [36] as well as anti-cancer drug resistance of lung cancer cells and ovarian carcinoma cells [37]. Moreover, another study also demonstrates 
that icariin exerts anti-tumor activity by upregulating the expression of ERS -related molecules (GRP78, CHOP, p-PERK and p-eIF2a), resulting in the inhibition of cell migration, adhesion and increase in apoptosis [38]. In the current study, we demonstrated that miR410 inhibition can suppress the expression of ERS-related genes, including CHOP, GRP94, Bip and the phosphorylation level of eIF $2 \alpha$. MiR-410 inhibition also reduced cell apoptosis via the upregulation of Bcl-2. However, ELRIN2 knockdown can abrogate the effects of miR-410 inhibitor on ERS signaling pathway, which was consistent with the findings in the previous study that ERLIN2 can modulate cell malignancy through the regulation the ERS pathway [7]. MiR-410 mimics had opposite effects on ERS pathway compared to that of miR410 inhibition. Taken together, our data indicate that miR-410 played anti-tumor activity by targeting ERLIN2 via the modulation of ERS pathway. However, the roles of ERS pathway in the chemotherapy resistance of breast cancer need to be detected further, which will be beneficial for exploring therapeutic and prognostic biomarker.

Accumulating evidence indicates that promoter hypermethylation accounts for the downregulation of miRNAs in a series of cancers [39]. For example, miR-375 is downregulated in HPV-16 positive cervical cancer cells partly due to promoter hypermethylation mediated by DNMT1 [40]. DNA methylation accounts for the downregulation of miR-152 in breast cancer cells which suppresses cell migration via the DNMT1/CDH1 loop [41]. Previous studies validate that DNA methylation is associated with hypoxia which will affect the activity of TET enzyme, DNMT1 $[42,43]$. The abnormal miRNA expression is related to tumor microenvironment, such as hypoxia [44, 45]. In our study, PCR showed that miR410 was increased after 5-Aza treatment, and MSP results indicated that miR-410 was hypermethylated in T47D and MCF-7 cells, respectively. However, miR-410 expression was not affected in the ER(-) cells after treated with 5-Aza. The data suggested that the deregulation of miR-410 might be due to the promoter methylation which might be not existed in ER(-) cells. However, we need further to investigate which methylation-related enzyme is involved and whether the abnormal miR-410 level is related to hypoxia of breast cancer.

In conclusion, this study indicate that miR-410 plays anti-tumor roles by directly targeting ERLIN2, acting as a tumor suppressor in ER(+) breast cancer. Moreover, the downregulation of miR-410 was related to promoter hypermethylation. All data suggest that miR-410 may serve as a potential therapeutic target for patients with $\mathrm{ER}(+)$ breast cancer, which will be a long way to be validated in the clinic experiment.

\section{Acknowledgements}

This study was supported by the National Natural Science Foundation of China (No. 81372855).

\section{Disclosure Statement}

The authors declare to have no conflict of interests.

\section{References}

1 Ferlay J, Soerjomataram I, Dikshit R, Eser S, Mathers C, Rebelo M, Parkin DM, Forman D, Bray F: Cancer incidence and mortality worldwide: sources, methods and major patterns in GLOBOCAN 2012. Int J Cancer 2015;136:E359-386.

2 DeSantis C, Ma J, Bryan L, Jemal A: Breast cancer statistics, 2013CA Cancer J Clin 2014;64:52-62.

3 Yager JD, Davidson NE: Estrogen carcinogenesis in breast cancer. N Engl J Med 2006;354:270-282. 


\section{Cellular Physiology Cell Physiol Biochem 2018;48:461-474 \begin{tabular}{l|l} 
DOI: 10.1159/000491777 & Ond Biochemistry \\
Published online: July 17, 2018 & $\begin{array}{l}\text { 2018 The Author(s). Published by S. Karger AG, Basel } \\
\text { www.karger.com/cpb }\end{array}$ \\
\cline { 2 - 3 }
\end{tabular}}

Wu et al.: the Act of MiR-410 on ERLIN2 in ER(+) Breast Cancer

-4 Yang ZQ, Streicher KL, Ray ME, Abrams J, Ethier SP: Multiple interacting oncogenes on the 8p11-p12 amplicon in human breast cancer. Cancer Res 2006;66:11632-11643.

5 Garcia MJ, Pole JC, Chin SF, Teschendorff A, Naderi A, Ozdag H, Vias M, Kranjac T, Subkhankulova T, Paish C, Ellis I, Brenton JD, Edwards PA, Caldas C: A 1 Mb minimal amplicon at 8p11-12 in breast cancer identifies new candidate oncogenes. Oncogene 2005;24:5235-5245.

6 Gelsi-Boyer V, Orsetti B, Cervera N, Finetti P, Sircoulomb F, Rouge C, Lasorsa L, Letessier A, Ginestier C, Monville F, Esteyries S, Adelaide J, Esterni B, Henry C, Ethier SP, Bibeau F, Mozziconacci MJ, Charafe-Jauffret E, Jacquemier J, Bertucci F, Birnbaum D, Theillet C, Chaffanet M: Comprehensive profiling of 8p11-12 amplification in breast cancer. Mol Cancer Res 2005;3:655-667.

7 Wang G, Liu G, Wang X, Sethi S, Ali-Fehmi R, Abrams J, Zheng Z, Zhang K, Ethier S, Yang ZQ: ERLIN2 promotes breast cancer cell survival by modulating endoplasmic reticulum stress pathways. BMC cancer 2012;12:225. Bartel DP: MicroRNAs: genomics, biogenesis, mechanism, and function. Cell 2004;116:281-297.

Yekta S, Shih IH, Bartel DP: MicroRNA-directed cleavage of HOXB8 mRNA. Science 2004;304:594-596. Ambros V: The functions of animal microRNAs. Nature 2004;431:350-355. Fang Y, Xue JL, Shen Q Chen J, Tian L: MicroRNA-7 inhibits tumor growth and metastasis by targeting the phosphoinositide 3-kinase/Akt pathway in hepatocellular carcinoma. Hepatology 2012;55:1852-1862.

12 Esquela-Kerscher A, Slack FJ: Oncomirs - microRNAs with a role in cancer. Nat Rev Cancer 2006;6:259-269.

13 Gatt ME, Zhao JJ, Ebert MS, Zhang Y, Chu Z, Mani M, Gazit R, Carrasco DE, Dutta-Simmons J, Adamia S, Minvielle S, Tai YT, Munshi NC, Avet-Loiseau H, Anderson KC, Carrasco DR: MicroRNAs 15a/16-1 function as tumor suppressor genes in multiple myeloma. Blood 2010; DOI: 10.1182/blood-2009-11-253294.

14 Volinia S, Calin GA, Liu CG, Ambs S, Cimmino A, Petrocca F, Visone R, Iorio M, Roldo C, Ferracin M, Prueitt RL, Yanaihara N, Lanza G, Scarpa A, Vecchione A, Negrini M, Harris CC, Croce CM: A microRNA expression signature of human solid tumors defines cancer gene targets. Proc Natl Acad Sci U S A 2006;103:2257-2261.

15 Iorio MV, Ferracin M, Liu CG, Veronese A, Spizzo R, Sabbioni S, Magri E, Pedriali M, Fabbri M, Campiglio M, Menard S, Palazzo JP, Rosenberg A, Musiani P, Volinia S, Nenci I, Calin GA, Querzoli P, Negrini M, Croce CM: MicroRNA gene expression deregulation in human breast cancer. Cancer Res 2005;65:7065-7070.

-16 Shi W, Bruce J, Lee M, Yue S, Rowe M, Pintilie M, Kogo R, Bissey PA, Fyles A, Yip KW, Liu FF: MiR-449a promotes breast cancer progression by targeting CRIP2 Oncotarget 2016;7:18906-18.

17 Yin K, Yin W, Wang Y, Zhou L, Liu Y, Yang G, Wang J, Lu J: MiR-206 suppresses epithelial mesenchymal transition by targeting TGF-beta signaling in estrogen receptor positive breast cancer cells. Oncotarget 2016; 7:24537-48.

18 Tan S, Ding K, Li R, Zhang W, Li G, Kong X, Qian PY, Lobie PE, Zhu T: Identification of miR-26 as a key mediator of estrogen stimulated cell proliferation by targeting CHD1, GREB1 and KPNA2 Breast Cancer Res. 2014;16:R40.

19 Ma L, Liu H, Xie Z, Yang S, Xu W, Hou J, Yu B: Ginsenoside Rb3 protects cardiomyocytes against ischemiareperfusion injury via the inhibition of JNK-mediated NF-kappaB pathway: a mouse cardiomyocyte model. PloS one 2014;9:e103628.

20 Zhang X, Ke X, Pu Q, Yuan Y, Yang W, Luo X, Jiang Q, Hu X, Gong Y, Tang K, Su X, Liu L, Zhu W, Wei Y: MicroRNA-410 acts as oncogene in NSCLC through downregulating SLC34A2 via activating Wnt/beta-catenin pathway. Oncotarget 2016;7:14569-14585.

-21 Mussnich P, Raverot G, Jaffrain-Rea ML, Fraggetta F, Wierinckx A, Trouillas J, Fusco A, D’Angelo D: Downregulation of miR-410 targeting the cyclin B1 gene plays a role in pituitary gonadotroph tumors. Cell cycle 2015;14:2590-2597.

22 Guo R, Gu J, Zhang Z, Wang Y, Gu C: MicroRNA-410 functions as a tumor suppressor by targeting angiotensin II type 1 receptor in pancreatic cancer. IUBMB life 2015;67:42-53.

23 Chen L, Zhang J, Feng Y, Li R, Sun X, Du W, Piao X, Wang H, Yang D, Sun Y, Li X, Jiang T, Kang C, Li Y, Jiang C: MiR-410 regulates MET to influence the proliferation and invasion of glioma. Int J Biochem Cell Biol 2012;44:1711-1717.

24 Shen J, Niu W, Zhou M, Zhang H, Ma J, Wang L, Zhang H: MicroRNA-410 suppresses migration and invasion by targeting MDM2 in gastric cancer. PloS one 2014;9:e104510.

25 Zhang X, Zheng L, Sun Y, Wang T, Wang B: Tangeretin enhances radiosensitivity and inhibits the radiationinduced epithelial-mesenchymal transition of gastric cancer cells. Oncol Rep 2015;34:302-310.

26 Li D, Yang Y, Zhu G, Liu X, Zhao M, Li X, Yang Q: MicroRNA-410 promotes cell proliferation by targeting BRD7 in non-small cell lung cancer. FEBS Lett 2015;589:2218-2223. 


\section{Cellular Physiology Cell Physiol Biochem 2018;48:461-474 \begin{tabular}{l|l} 
DOI: 10.1159/000491777 & and Biochemistry \\
Published online: July 17, 2018 & $\begin{array}{l}\text { O 2018 The Author(s). Published by S. Karger AG, Basel } \\
\text { www.karger.com/cpb }\end{array}$
\end{tabular}}

Wu et al.: the Act of MiR-410 on ERLIN2 in ER(+) Breast Cancer

-27 Wang Y, Fu J, Jiang M, Zhang X, Cheng L, Xu X, Fan Z, Zhang J, Ye Q, Song H: MiR-410 is overexpressed in liver and colorectal tumors and enhances tumor cell growth by silencing FHL1 via a direct/indirect mechanism. PLoS One 2014;9:e108708.

28 Liu D, Zhang N, Zhang X, Qin M, Dong Y, Jin L: MiR-410 Down-Regulates the Expression of Interleukin-10 by Targeting STAT3 in the Pathogenesis of Systemic Lupus Erythematosus. Cell Physiol Biochem 2016;39:303315.

29 Zhang X, Cai J, Zheng Z, Polin L, Lin Z, Dandekar A, Li L, Sun F, Finley RL, Jr., Fang D, Yang ZQ Zhang K: A novel ER-microtubule-binding protein, ERLIN2, stabilizes Cyclin B1 and regulates cell cycle progression. Cell Discov 2015;1:15024.

- 30 Cheng C, Fu X, Alves P, Gerstein M: mRNA expression profiles show differential regulatory effects of microRNAs between estrogen receptor-positive and estrogen receptor-negative breast cancer. Genome Biol 2009;10:R90.

-31 Adams BD, Claffey KP, White BA: Argonaute-2 expression is regulated by epidermal growth factor receptor and mitogen-activated protein kinase signaling and correlates with a transformed phenotype in breast cancer cells. Endocrinology 2009;150:14-23.

-32 Liu N, Yang HL, Wang P, Lu YC, Yang YJ, Wang L, Lee SC: Functional proteomic analysis revels that the ethanol extract of Annona muricata L. induces liver cancer cell apoptosis through endoplasmic reticulum stress pathway. J Ethnopharmacol 2016;189:210-217.

-33 Banerjee A, Ahmed H, Yang P, Czinn SJ, Blanchard TG: Endoplasmic reticulum stress and IRE-1 signaling cause apoptosis in colon cancer cells in response to andrographolide treatment. Oncotarget 2016;7:4143241444.

-34 Sanz-Pamplona R, Aragues R, Driouch K, Martin B, Oliva B, Gil M, Boluda S, Fernandez Pl, Martinez A, Moreno V, Acebes JJ, Lidereau R, Reyal F, Van de Vijver MJ, Sierra A: Expression of endoplasmic reticulum stress proteins is a candidate marker of brain metastasis in both ErbB-2+ and ErbB-2- primary breast tumors. Am J Pathol 2014;179:564-579.

-35 Dollins DE, Warren JJ, Immormino RM, Gewirth DT: Structures of GRP94-nucleotide complexes reveal mechanistic differences between the hsp90 chaperones. Mol Cell 2007;28:41-56.

-36 Zheng HC, Takahashi H, Li XH, Hara T, Masuda S, Guan YF, Takano Y: Overexpression of GRP78 and GRP94 are markers for aggressive behavior and poor prognosis in gastric carcinomas. Hum Pathol 2008;39:1042-1049.

-37 Zhang L, Wang SF, Wang T, Wang Y, Wang J, Jiang L, Li S, Hu X, Wang Q: Upregulation of GRP78 and GRP94 and its function in chemotherapy resistance to VP-16 in human lung cancer cell line SK-MES-1 C a n c e r Invest 2009;27:453-458.

-38 Fan C, Yang Y, Liu Y, Jiang S, Di S, Hu W, Ma Z, Li T, Zhu Y, Xin Z, Wu G, Han J, Li X, Yan X: Icariin displays anticancer activity against human esophageal cancer cells via regulating endoplasmic reticulum stressmediated apoptotic signaling. Sci Rep 2016;6:21145.

-39 Shen G, Li X, Jia YF, Piazza GA, Xi Y: Hypoxia-regulated microRNAs in human cancer. Acta Pharmacol Sin 2013;34:336-341.

40 Liu S, Song L, Yao H, Zhang L, Xu D, Gao F, Li Q: MiR-375 Is Epigenetically Downregulated by HPV-16 E6 Mediated DNMT1 Upregulation and Modulates EMT of Cervical Cancer Cells by Suppressing IncRNA MALAT1 PLoS One 2016;11:e0163460.

41 Sengupta D, Deb M, Rath SK, Kar S, Parbin S, Pradhan N, Patra SK: DNA methylation and not H3K4 trimethylation dictates the expression status of miR-152 gene which inhibits migration of breast cancer cells via DNMT1/CDH1 loop. Exp Cell Res 2016;346:176-187.

-42 Thienpont B, Steinbacher J, Zhao H, D’Anna F, Kuchnio A, Ploumakis A, Ghesquiere B, Van Dyck L, Boeckx B, Schoonjans L, Hermans E, Amant F, Kristensen VN, Koh KP, Mazzone M, Coleman ML, Carell T, Carmeliet P, Lambrechts D: Tumour hypoxia causes DNA hypermethylation by reducing TET activity. Nature 2016;537:6368.

43 McDonnell FA-O, Irnaten M, Clark AF, O’Brien CJ, Wallace DM: Hypoxia-Induced Changes in DNA Methylation Alter RASAL1 and TGFbeta1 Expression in Human Trabecular Meshwork Cells. PLoS One 2016;11:e0153354.

44 Rupaimoole R, Calin GA, Lopez-Berestein G, Sood AK: miRNA Deregulation in Cancer Cells and the Tumor Microenvironment. Cancer Discov 2016;6:235-246.

45 Huang CX, Zhu Y, Duan Gl, Yao JF, Li ZY, Li D, Wang QQ: Screening for MiRNAs related to laryngeal squamous carcinoma stem cell radiation. Asian Pac J Cancer Prev 2013;14:4533-4537. 